\title{
Pregnancy related lumbo-pelvic pain: a prevalence study in a tertiary institute in Pondicherry, India
}

\author{
R. Arunmozhimaran Vijayababu', Vijaya Koothan ${ }^{2} *$, D. Krishna Prabhu', Aakash Putta ${ }^{1}$
}

\begin{abstract}
${ }^{1}$ Department of Orthopaedics, ${ }^{2}$ Department of Obstetrics \& Gynaecology, Arupadai Veedu Medical College \& Hospital, Kirumampakkam, Pondicherry, India
\end{abstract}

Received: 31 January 2016

Revised: 03 March 2016

Accepted: 08 March 2016

\author{
*Correspondence: \\ Dr. Vijaya Koothan, \\ E-mail: vijayaceline7@gmail.com
}

Copyright: (C) the author(s), publisher and licensee Medip Academy. This is an open-access article distributed under the terms of the Creative Commons Attribution Non-Commercial License, which permits unrestricted non-commercial use, distribution, and reproduction in any medium, provided the original work is properly cited.

\begin{abstract}
Background: Pregnancy related low lumbo- pelvic pain (PLPP) is a common complaint that occurs in 60-70\% of pregnancies. It can begin at any point during pregnancy. Although most cases are mild, approximately one third of women experience severe pain. The aim of the study was to determine the prevalence of PLPP among antenatal mothers in a tertiary care institute in Pondicherry, India and assess the disability caused therein.

Methods: 202 consecutive pregnant women screened for PLPP in the antenatal clinic in Aarupadi Veedu Medical College Hospital Pondicherry, India by questionnaire and assessment of lumbar spine by Mckenzie protocol 2003 and pelvis by specific physical examination for evaluation of low back ache. 31 women had low back ache out of 202 who took part in screening procedure in the antenatal clinic at tertiary care institute to study the point prevalence in the given population disability was assessed by Oswestry disability index scoring questionnaire.

Results: Prevalence of PLPP was as low as $15.35 \%$ compared to an average of $45 \%$ in European studies. PLBP was the dominant clinical pattern $54.84 \%$, PLPP plus PPGP combined was $41.94 \%$ and PPGP was a very rare pattern $3.23 \%$ Oswestry disability index (ODI) in this study suggests scores upto 30 (mild to moderately disability).

Conclusions: PLPP is a disabling palpable clinical problem less recognised in India and most often dismissed as part of pregnancy. Sensitization of obstetrician and the orthopaedician to interact in a multidisciplinary approach would address the problem effectively.
\end{abstract}

Keywords: Pregnancy related lumbo-pelvic pain, Pregnancy related low back ache, Gestation related low backache

\section{INTRODUCTION}

$45 \%$ of pregnant women and $25 \%$ women post-partum suffer from lumbo-pelvic pain associated with pregnancy. ${ }^{1}$ Pregnancy related low back pain (PLBP) is a common complaint in pregnancy in European studies, that occurs in $60-70 \%$ of pregnancies. ${ }^{2}$ It can be defined as pain between the $12^{\text {th }}$ rib and the gluteal folds/pubic symphysis during the course of pregnancy. It can begin at any point during pregnancy. Although most cases are mild, approximately one third of women experience severe pain. Antepartum population is at high risk for falls, comparable to the geriatric population. One fourth of them would have experienced a fall and one third of them would have had two or more times during pregnancy. Third trimester, 28 weeks has the highest rate of falls. ${ }^{3}$

Pregnancy related low back pain PLBP has been recognized even as early as $400 \mathrm{BC}$ by Hippocrates. He recognized widening of symphysis pubis during first parturition remained permanent and facilitated child birth subsequently. Snelling stated that this relaxation was a physiological preparation for delivery. Vasalius 
recognized synovial fluid and swelling of joints. Goeschke in 1912 reported increased tissue, hyperemia and hypertrophy of ligaments. In 1928 more than $20 \mathrm{~mm}$ of joint space was considered as marked diastasis by Bren and Weiranch, in 1932 Hayman Lundquis observed that widening of symphysis pubis starts in early pregnancy, and continues up to 3 to 4 months before parturition. ${ }^{4}$

Later, Ostgaard et al, set the criteria for the differentiation between these two entities. It has been estimated that about $50 \%$ of pregnant women will suffer from some kind of low back pain at some point during their pregnancies or during the postpartum period. ${ }^{5}$

Pelvic girdle pain has come to be recognized as a distinct only in the last few decades. The aetiology of PLBP has been poorly understood. It has been proposed that due to increased weight gain in pregnancy, shifting of the centre of gravity of the body anteriorly and stretching the abdominal muscles to accommodate the enlarging uterus causes muscle fatigue.

Despite a familiarity of the entity since centuries, little has been found to be concrete in the literature, with a wide range of variation of prevalence, with different methodology of studies, classification and nomenclature and varying degrees of disability. Most studies reported were from the Europe where the population are aware of the entity and health care providers recognize the condition as associated with pregnancy in their management. Very few studies have been reported in Asia and still fewer in India. We embarked on the estimation of the problem and assess the disability therein.

Although a perceptive problem among pregnant women, women barely take cognisance of it and hardly seek medical help. Women in India are inclined to bear pain to the point of unbearability and put off any consideration for medical consultation, as it is often conveniently contrived as part and parcel of pregnancy issues and dismissed readily.

The aim of the study was to determine the prevalence and disability caused by low back ache in pregnant women among women attending antenatal clinic in AVMCH Pondicherry, India.

\section{METHODS}

Observational study, recruiting consecutive 202 pregnant women attending antenatal clinic in AVMC \& $\mathrm{H}$ Pondicherry, India from September 2015 to January 2016. The women received written and verbal information about the study before giving consent and study was conducted in fulfillment of ethical consideration of the institute ethics committee.

Pregnant women more than 12 weeks of gestation attending antenatal clinic /ward or orthopaedics outpatient department were recruited in the study. Systemic locomotor system disease, history of spinal problems in the previous two months; or a history of fracture, neoplasm, or previous spinal, pelvic, or femur surgery were excluded. Non orthopaedic causes for low back pain were excluded by a questionnaire by screening for gastrointestinal, urological, gynaecological obstetrical causes of low back ache.

Past medical history experienced symptoms, limitations in activities, and movement disabilities were elicited. Assessment to differentiate between the lumbar spine and pelvic dysfunction were done. Physical examination was done, with wedge under the right buttock in left 15 degree lateral position avoiding supine hypotension syndrome. Examination included posture and gait, neurologic screening to rule out underlying pathology, range of motion, muscle power tests, palpation, muscle length tests, and assessment of joint mobility. Specific tests included; (1) distraction test; (2) posterior pelvic pain provocation test; (3) Gaenslen's test; (4) compression test; (5) sacral thrust; (6) The active straight leg raise test. The mechanical assessment of the lumbar spine was based on the MDT protocol (McKenzie and May2003). After the examination, the women were classified into three groups based on the criteria decided on before the examination.

\section{The criteria for being assigned PPGP group}

Stabbing, shooting, dull or burning. Intermittent precipitated by prolonged sustained posture, walking, sitting, standing, twisting, climbing, unequal weight bearing on legs, turning in bed. Pain experienced distal to L5, pain in sacroiliac joints, between the posterior iliac crest and the gluteal fold, with or without radiation in the posterior thigh and calf and with or without pain in the symphysis. Pain reproducible by at least two out of the five pelvic pain provocation tests (two tests bilaterally). ${ }^{6}$ No centralization or peripheralisation phenomenon and no change in lumbar pain or the range of motion from repeated movements according to the MDT classification Onset of PGP was required to be in relation to pregnancy. PGP has been further classified into five groups anterior, posterior, Both $\mathrm{B} / \mathrm{L}$ SIJ, miscellaneous, complete pelvic girdle syndrome with pain in all 3 pelvic joints. $^{6}$

\section{The criteria for being assigned to the lumbar pain group}

Pain experienced in the lumbar region, with or without radiation to the leg reproducible pain and/ or a change in the range of motion from repeated movements or different positions of the lumbar spine or an experience of centralisation and/or peripheralisation during examination fewer than two positive pelvic pain provocation tests. 


\section{The criteria for being assigned to the combined pain group}

Pain in the lumbar region as well as between the posterior iliac crest and the gluteal fold, with or without radiation in the posterior thigh and calf, and with or without pain in the symphysis, two or more positive pain provocation tests pain and/or a change in the range of motion from repeated movements or different positions of the lumbar spine, or experienced centralisation and/or peripheralisation. After classification, the women were referred for treatment.

In the event of vaginal bleeding, dizziness/feeling faint, shortness of breath, chest pain, headache, muscles weakness, calf pain or swelling, uterine contractions decreased fetal movement, vaginal fluid leakage, neurological involvement, the evaluation with be different. Outcome measures were recorded as disability score assessments with Oswestry disability questionnaire and Mckenzie lumbar assessment form.

\section{RESULTS}

Total of 202 consecutive pregnant mothers participated in the study, all were accessible for documentation. 31/202 $(15.35 \%)$ women were found to suffer from low back ache related to pregnancy, PPGP was $1 / 31$ (3.23\%), PLBP-17/31 (54.84\%), combined (PLBP and PPGP) were $13 / 31(41.94 \%)$.

2/33 participants with history of low back ache had history of previous trauma that was excluded from the study. All 31 participants with low back ache in pregnancy consent to and underwent further evaluation by questionnaires, physical examinations uneventfully. All women were housewives. Distraction test was negative in all women. None of the women were involved with strenuous work in pregnancy, they had assistance for rigorous domestic mundane work too.

In our study lumbo-pelvic pain presented in 1/31, 9/31, 19/31- I, II, III trimesters respectively. The third trimesters being the commonest time of presentation.

The pregnant women classified as suffering from pregnancy related lumbar pain fulfilled all the criteria of PLBP characteristics, but women classified as PPGP, and combined PPGP plus PLBP fulfilled all other criteria mentioned in the methods mentioned above except positive pelvic provocation test. The single case of PPGP failed to demonstrate even one provocation test to be positive, and in the combined group, provocation test was positive in only 3 out of 13 cases.

Oswestry disability scoring assessment suggests, 66\% had mild disability score upto $20,22 \%$ had moderate disability - (21-30), 12\% of PLPP had no disability .

Table 1: Demographics of participants.

\begin{tabular}{|llll|}
\hline $\begin{array}{l}\text { Patient } \\
\text { character } \\
\text { istics }\end{array}$ & PPGP & PLBP & $\begin{array}{l}\text { PPGP,PLBP } \\
\text { combined }\end{array}$ \\
\hline & 1 & $17 / 31$ & $13 / 31$ \\
\hline Age & 33 & $\begin{array}{l}23.94 \text { yrs, } \\
25,25\end{array}$ & $23.85,23,21$ \\
\hline parity & Multipara & $\begin{array}{l}\text { Primi-5/17 } \\
(29.4 \%)\end{array}$ & $\begin{array}{l}\text { Primi-4/13 } \\
(30.77 \%)\end{array}$ \\
& & $\begin{array}{l}\text { multipara- } \\
\text { multi-9/13 }\end{array}$ & $\begin{array}{l}(2 / 17 \\
\end{array}$ \\
& & $(70.6 \%)$ & \\
\hline
\end{tabular}

Table 2: Pain characteristics.

\begin{tabular}{|c|c|c|c|}
\hline $\begin{array}{l}\text { History of low back ache in } \\
\text { previous pregnancy }\end{array}$ & $1 / 1$ & $3 / 17(17.64 \%)$ & $4 / 13(30.76 \%)$ \\
\hline Onset of pain & from conception & $\begin{array}{l}\text { range }-53-265 \\
\text { mean } 164.88 \text { days } \\
\text { median } 161 \\
\text { mode }-116,259 \text { days }\end{array}$ & $\begin{array}{l}\text { from conception }-273 \text { days } \\
\text { mean }-181.53 \text { days } \\
203 \\
\text { mode from conception }\end{array}$ \\
\hline Duration of pain & 90 days & $\begin{array}{l}1-180 \text { days } \\
51.9 \text { days } \\
7 \\
7\end{array}$ & $\begin{array}{l}\text { range } 3-365 \text { days } \\
64 \\
10 \\
7\end{array}$ \\
\hline Intensity & mild & 3/17, VAS -2-4 & $4 / 13$, VAS score -2 \\
\hline ODI score & 0 & $\begin{array}{l}\text { 9/17 (52.94\%) - Mild } \\
\text { 2/17 (11.76\%) - Moderate }\end{array}$ & $\begin{array}{l}\text { 9/13 }(69.23 \%) \text { - Mild } \\
\text { 2/13 (15.38\%) - Moderate }\end{array}$ \\
\hline Sleep disturbance & 0 & 5/17 29.41\% - Mild & 6/13 (46.15\%) - Mild \\
\hline Sexual difficulties & 0 & 1/17 (5.88\%) - Mild & 1/13 (7.69\%) - Mild \\
\hline
\end{tabular}

ODI -Oswestry disability 


\section{DISCUSSION}

In our study the overall prevalence of pregnancy related low back pain PLPP was $15.35 \%$. PLBP was the predominant clinical pattern observed here. PPGP was 1/31 (3.23\%), PLBP-17/31 (54.84\%), combined (PLBP

and PPGP) were 13/31 (41.94\%). In our study combined PLBP has been the dominant clinical pattern unlike in the western studies. In European studies, PPGP has been reported to be half of the observed clinical presentation of PLPP.

The review of literature of large studies offers little consistency in the epidemiology of PPGP. This large variation in prevalence of PPGP and PLBP could be attributed to poor understanding and definition of this entity and inconsistent classification and terminologies of PLPP. The design of the study and the diagnostic criteria used have been diverse and non-uniform and the type of studies like retrospective and prospective studies with variable levels of evidence. Several studies in literature used self reported questionnaire and many authors did not insist on physical examination for diagnosis.

Wu et al in a review of 28 studies reported $45 \%$ of all pregnant women \& $25 \%$ of all post-partum women suffered PGP +/or PLBP. ${ }^{1}$ Larson et al in a case control study of 1600 pregnant women reported a prevalence of PLPP to be $14 \%$. He considered the women to have pelvic girdle relaxation if at least two activities of daily living, like turning in bed, walking, lifting light load, getting up from chair, climbing stairs. The participants were followed up during and after pregnancy by interview and clinical examination at 16, 20, 30, 33, 38 and 40 weeks of pregnancy. The prevalence in this study was similar to our findings. However, as Larsen et al only examined $14.8 \%$ of the pregnant women included in the study. $^{7}$

Wang et al, from Connecticut USA in a cross sectional study screening 950 pregnant women with self reported questionnaires, during pregnancy, reported a prevalence of $58.5 \%$ low back pain in pregnancy. Wang has not given any clear definition of symptoms of low back pain in pregnancy. ${ }^{8}$ In 1962 , Walde was the first to recognise PPGP and LPP as different patterns of PPLP. ${ }^{9}$ The term PPGP was coined in the recent years in 2005. ${ }^{9}$ Ostgaard introduced the pelvic provocation test in diagnosis of PPGP. Systemic and physical examination is a recent trend followed subsequent to Ostgaard's introduction of pelvic provocation test. Previously this distinction was not acknowledged. Many studies fail to discriminate different patterns of clinical presentation of low back ache namely - PPGP, LBP, and combined. ${ }^{4}$

Very few studies in literature distinguish between PPGP and PLBP. Ostgaard et al from Sweden in prospective cohort study between 12 weeks of pregnancy and 1 year after delivery at 4 weeks interval screened 855 pregnant women for lumbar pain using self-reported questionnaire pain mapping and visual analogue scale found a point prevalence of 25\% PLPP. He differentiated lumbar back pain from PPGP specific to pregnancy. The point prevalence (the number of pregnant women at week 33 with PPGP) was $20.1 \%$. Based on above described studies, point prevalence of women suffering from PPGP during pregnancy is close to $20 \%$. $^{5}$

Gupta et al reported the prevalence of PPGP in north Indian population to be $60 \% .^{10}$ This study has taken into account PPGP and combined PPGP and PLBP entity, but makes no mention of presentation of PLBP as separate presentation.

Albert et al from Denmark in a case controlled study found pelvic joint pain in 405/1789 of pregnant women, with questionnaire method and physical examination between 33 weeks of pregnancy and 24 months post partum. He reported prevalence of PPGP to be $22.6 \% .^{11}$

Kristiansson from Sweden screened 200 women in a prospective cohort study in early pregnancy attending antenatal clinic using questionnaires, pain sketches, and visual analogue scales and disability ratings, between 12 weeks of pregnancy and 12 weeks after delivery. Evaluating at 12, 24, 36 weeks antenatally and 12 weeks postpartum, reported $76.4 \%$ period prevalence of low back ache ${ }^{12}$.

Among a review of 19 studies reported by Kristiansson prevalence reports by questionnaire alone ranged from 24-89.9\% among the studies where pain was undefined. In two of nineteen studies by questionnaires method alone reported PLPP to be 56\% and 49\%. In 4/19 studies involving both questionnaire method and physical examination, prevalence of PPGP was found to be 14$44.8 \%{ }^{13} 13 / 19$ studies failed to define the complaint.

Gutke et al in a cohort study of 313 pregnant women, (reported) with Oswestry questionnaire and physical examination, questionnaire and physical examination, demographic details and studied the difference between PGP, LPP . He reported PGP - LPP - 194/313 (61.98\%), PPGP-(54\%), LP-(17\%), $\quad$ PPGP+LP-(29\%). ${ }^{14} \quad 61$ clinicians across 5 regions in Spain recruited 1158 women with pregnancy of 35 (31-38) weeks. The prevalence reported was PLPP $71.3 \%$, PLBP, PPGP $46.2 \%$ and $64.2 \%$ respectively. ${ }^{15}$

Berg et al performed a prospective study in which 862 pregnant women completed a questionnaire in weeks 20 , 30 , and 35 of gestation. Of these women, $49 \%$ reported that they experienced SIJ pain at some time during the pregnancy, i.e. the cumulative incidence (defined as how many women reported by questionnaire at 3 different times during their pregnancy that they felt pain in their pelvic area). However, only women entitled to sick leave from work $(9 \%)$ underwent clinical examination. ${ }^{16}$ 
Variation of reported prevalence could be attributed to various factors and variables like severity of symptoms, health seeking behaviour of a population. Outpatient selfreported data exaggerated the frequency, while diagnosed by doctors who consider the symptoms deemed necessary for treatment the estimates were less by $20 \%$. Other variables include timing of symptoms in the course of pregnancy, during or after pregnancy, whether point prevalence or cumulative period prevalence studied, recognizing and distinguishing the indistinct clinical patterns, inconsistent nomenclature, the method of diagnosis, whether questionnaire alone was used or physical examination followed, contribute to the spectrum wide prevalence estimates in various studies. PGP is included in LBP. The low back ache sometimes lacks definition of localisation of pain and even one clinical pattern may progress into another pattern changing its characteristics from one to the other. ${ }^{13}$ Increased awareness of low back ache caused by pregnancy by the population in Scandinavian countries and familiarity with the care givers draw attention and pronounce the prevalence in European studies. It has its impact on socioeconomic and absenteeism from work in the west. Patients ask for early termination of pregnancy, induction or caesarean section.

Wu et al in study of associated factors in analysis of 34 studies identified strenuous work, previous LBA, previous history of PGP, PLBP as risk factors to PLPP. ${ }^{1}$ Factors not affecting PLPP were use of oral contraceptive pills, spacing of pregnancy, BMI, smoking, age, epidural/spinal anaesthesia, analgesic technique.

In our study compared to European studies prevalence of PLPP is far less $(15.35 \%)$.The rough estimation of true prevalence is $45 \%$ of all pregnancies considering large prospective studies in Europe. 25\% of all pregnant women, $5 \%$ of all post partum women require medical help. Disability in lumbar pelvic pain during pregnancy ranges from $21-81 \%$ median $28 \% .^{17}$

It has been proposed that due to increased weight gain in pregnancy, shifting of the centre of gravity of the body anteriorly and stretching the abdominal muscles of accommodation the enlarging uterus causes muscle fatigue. In India the cultural habits of squatting, sleeping on flat firm surface is common; This maintains the spine in physiological position and may contribute to spontaneous realignment of minor malalignments of lumbo-pelvic joints. Socio political influences of government schemes and incentives Tamil Nadu, India obviate the need for strenuous domestic work such as grinding, pounding which are risk factors in precipitating low back pain.

Inspite of progressively increasing literature diagnosis of PLPP lacks gold standard criteria.

We could not classify and put into water-tight compartments of PGPP, PLBP, and PGPP, PLBP combined patterns. However pain was perceptible, mildly disabling and overlapping. All cases of PPGP or PPGP PLPP combined, failed to demonstrate a positive provocation test or two. This study was conducted on a limited number of sample sizes. The prevalence in our study does not reflect the estimate of problem in the general population as it represents only a part of the socio economic strata. The socio-economic, cultural and psychological components in pain perception and threshold could influence the prevalence.

Pregnancy related lumbar pelvic pain has been considered as a "hysterical epidemic", normal discomfort of pregnancy, and as a severe disability. It attracts attention frustration during and after delivery, anxiety about outcome in pregnancy delivery. Delineating the clinical patterns and containing it in classification is still elusive, despite considerable literature.

PLPP is ubiquitous, occurs in all countries. PLPP is no trivial symptom, it could be the beginning of a lifelong chronic back pain, and it may be disabling during pregnancy and may be relieved thereafter. Diagnosis could be elusive and is by exclusion. Invasive procedures are not recommended in pregnancy. Conservative management is the dictum in pregnancy. Analgesics, physiotherapy and specific exercise and postural adaptations are advisable.

Prospective studies involving large number of participants, randomized controlled trials would make statistical evidence strong. Further evaluation of the mechanism, investigations and management could help address the issue.

\section{CONCLUSION}

Prevalence of PLPP in this study group has been as low as $15.35 \%$ unlike in the western studies. PLBP was the dominant clinical pattern. PPGP was remarkably very low $3.23 \%$ and the findings were atypical with negative provocation tests. Disability assessment by Oswestry disability index suggests mild to moderate disability.

Pregnancy related low back ache deserves to be recognized as an entity both by the patients, relatives, and by particularly the health care providers. PLPP is a palpable problem faced by pregnant women, more often shoved under the carpet as insignificant or part and part of obstetric venture.

Clear understanding of low back ache,its recognition of clinical patterns, and well defined criteria for definition, and guidelines to management would afford optimal and appropriate treatment. Indiscriminate advice on postures which may be appropriate to one clinical pattern may aggravate another.

A multidisciplinary approach to pregnant mothers and enhancing the awareness among the community would 
address the problem and allay the socio economic detriments namely disability, absenteeism, limitation of sexual activity and discord in family life of a woman in reproductive age group.

\section{ACKNOWLEDGEMENTS}

We wish to thank Compulsory rotatory resident interns S. Siva Prasanna, B. Saranya, J. Varunya, A.S. Pradeep, Abilasha, and Uday Kumar for their assistance in data collection .

Funding: No funding sources

Conflict of interest: None declared

Ethical approval: The study was approved by the Institutional Ethics Committee

\section{REFERENCES}

1. Wu WH, Meijer OG, Uegaki K, Mens JMA, Van Dieën JH, Wuisman PIJM, ÖstgaardHC. Pregnancyrelated pelvic girdle pain (PPP), I : Terminology, clinical presentation, and prevalence. European Spine Journal. 2004;13(7):575-89.

2. Gutke A, Kjellby-Wendt G, Öberg B. The inter-rater reliability of a standardized classification system for pregnancy-related lumbopelvic pain. Manual therapy. 2010:15;13-8.

3. Dunning K1, Lemasters G, Bhattacharya A, et al. A major public health issue: the high incidence of falls during pregnancy. Matern Child Health J. 2010;14(5):720-5.

4. Bastiaanssen JM, de Bie RA, Bastiaenen $\mathrm{CH}$, Essed GG, van den Brandt PA et al, A historical perspective on pregnancy-related low back and/or pelvic girdle pain. Eur J ObstetGynecolReprod Biol. 2005;120:3-14.

5. Ostgaard HC, Andersson GB, Karlsson K, et al. Prevalence of back pain in pregnancy. Spine (Phila Pa 1976). 1991;16(5):549-52.

6. Laslett M1, Aprill CN, McDonald B, Young SB et al, Diagnosis of sacroiliac joint pain: validity of individual provocation tests and composites of tests. Man Ther. 2005;10(3):207-18.

7. Larsen EC, Wilken-Jensen C, Hansen A, Jensen DV, Johansen S, Minck H, Wormslev M, Davidsen M,
Hansen TM, et al. Symptom-giving pelvic girdle relaxation in pregnancy. I: Prevalence and risk factors. Acta Obstet Gynecol Scand. 1999;78(2):10510 .

8. Wang SM, Dezinno P, Maranets I, Berman MR, Caldwell-Andrews AA, Kain ZN, et al. Low back pain during pregnancy: prevalence, risk factors, and outcomes. Obstet Gynecol. 2004;104(1):65-70.

9. Walde J. Obstetrical and gynaecological back and pelvic pains, especially those contracted during pregnancy. Obstet Gynecol Scand. 1962;2:11-53.

10. Gupta M. Prevalence of pregnancy related pelvic girdle. Indian Journal of Obstetrics and Gynecology Research. 2014;1(1):16-23.

11. Albert H, Godskesen M, Westergaard J, et al. Prognosis in four syndromes of pregnancy-related pelvic pain. Acta Obstet Gynecol Scand. 2001;80(6):505-10.

12. Kristiansson P, Svardsudd K, Schoultz von B. Back pain during pregnancy: a prospective study. Spine. 1996;21:702-9.

13. Kristiansson P, Svardsudd, K. Discriminatory power of tests applied in back pain during pregnancy. Spine. 1996;21:2337-43.

14. Gutke A, Ostgaard HC, Oberg B, et al. Pelvic girdle pain and lumbar pain in pregnancy: a cohort study of the consequences in terms of health and functioning.Spine (Phila Pa 1976). 2006;31(5):E14955.

15. Kovacs FM, Garcia E, Royuela A, González L, Abraira V. Prevalence and factors associated with low back pain and pelvic girdle pain, during pregnancy - a multi centred study conducted in the Spanish national health service, spine (Phila. Pain 1976). 2012;1:37(17):1516-33.

16. Berg G1, Hammar M, Möller-Nielsen J, Lindén U, Thorblad J, et al. Low back pain during pregnancy. Obstet Gynecol. 1988;71(1):71-5.

17. Padua L, Caliandro P, Aprile I. Back pain in pregnancy: 1-year follow-up of untreated cases. Eur Spine J. 2005;14(2):151-4.

Cite this article as: Vijayababu AR, Koothan V, Prabhu DK, Putta A. Pregnancy related lumbo-pelvic pain - a prevalence study in a tertiary institute in Pondicherry, India. Int J Reprod Contracept Obstet Gynecol 2016;5:1124-9. 